\title{
Heterogeneous chromosomal aberrations in intraductal breast lesions adjacent to invasive carcinoma
}

\author{
Michaela Aubele ${ }^{\mathrm{a}, *}$, Margaret Cummings ${ }^{\mathrm{b}}$, \\ Axel Walch ${ }^{\text {a }}$ Horst Zitzelsberger ${ }^{c, d}$, Jörg Nährig ${ }^{\mathrm{e}}$, \\ Heinz Höfler ${ }^{\mathrm{a}, \mathrm{e}}$ and Martin Werner ${ }^{\mathrm{e}}$

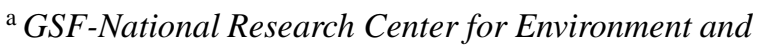 \\ Health, Institute of Pathology, Neuherberg, Germany \\ ${ }^{\mathrm{b}}$ Department of Pathology, University of Queensland, \\ Medical School, Queensland, Australia \\ ${ }^{\mathrm{c}}$ GSF-National Research Center for Environment and \\ Health, Institute of Radiobiology, Neuherberg, \\ Germany \\ ${ }^{\mathrm{d}}$ Ludwig Maximilians Universität, Institute of \\ Radiobiology, Munich, Germany \\ ${ }^{\mathrm{e}}$ Technische Universität München, Institute of \\ Pathology, Munich, Germany
}

Received 29 November 1999

Accepted 14 March 2000

There is evidence that breast cancer is a heterogeneous disease phenotypically as well as molecular biologically. So far, heterogeneity on the molecular biological level has not been investigated in potential precursor lesions, such as ductal hyperplasia (DH) and ductal carcinoma in situ (DCIS). In this study we applied comparative genomic hybridization (CGH) to formalin-fixed, paraffin-embedded breast tissue with $\mathrm{DH}$ and DCIS, adjacent to invasive ductal carcinoma (IDC), to screen these potential precursor lesions for whole genomic chromosomal imbalances. Laser-microdissection was used to select pure cell populations from the sections. Isolated DNA was amplified by degenerate oligonucleotide primed PCR (DOP-PCR) and further processed for CGH analysis.

Investigating multiple samples $(n=25)$ from four patients we found an average of $5.6 \pm 0.9$ (mean \pm SEM) chromosomal imbalances already present in DH. In the twelve DCIS lesions an average of $10.8( \pm 0.9)$ aberrations was identified with $14.8( \pm 0.8)$ aberrations in the four adjacent IDC

\footnotetext{
*Correspondence to: Dr Michaela Aubele, GSF-Forschungszentrum, Institut für Pathologie, Ingolstädter Landstraße 1, 85764 Neuherberg, Germany. Tel.: +49 893187 4132; Fax: +49 893187 3360; E-mail: aubele@gsf.de.
}

lesions. The increasing number of chromosomal changes in parallel with the histopathological sequence corroborate the hypothesis, that the carcinomas may have developed through a sequential progression from normal to proliferative epithelium and eventually into carcinoma. However, heterogeneous results were identified in the multiple samples per entity from the same patient, demonstrated mainly in the DCIS samples in the chromosomal regions $6 \mathrm{p}, 9 \mathrm{p}, 11 \mathrm{q}, 16 \mathrm{p}$ and $17 \mathrm{q}$, in the DH samples by $3 p, 16 p$ and $17 q$. This heterogeneous findings were most pronounced within the $\mathrm{DH}$ and was less in the DCIS and IDC samples. The only aberration consistently found in all samples - even in all DH samples - was amplification of the $20 \mathrm{q} 13$ region.

Our results demonstrate, that the applied combination of laser-microdissection, DOP-PCR and CGH, may serve to analyse breast carcinogenesis pathways in suitable histological material. However, so far, it is unclear how to handle heterogeneous results and these make identification of relevant changes more difficult. Setting a threshold and valuating only those chromosomal changes which are present in a majority of samples may be one possibility. This involves however, the risk that infrequent but possibly significant aberrations may be missed.

Figures on http://www.esacp.org/acp/2000/20-1/aubele.htm.

\section{Introduction}

Carcinoma of the breast is thought to evolve through a sequential progression from normal to proliferative epithelium and eventually into carcinoma, but molecular biological data supporting this progression are limited [17]. Proliferative breast lesions are regarded as benign disorders, yet epidemiologic studies indicate that they are associated with a significantly increased risk of developing breast cancer [21]. Based on such studies, a model of breast tumorigenesis has been proposed in which normal epithelium becomes proliferative (ductal hyperplasia, DH) and then, through an accumulation of molecular abnormalities, evolves into ductal carcinoma in situ (DCIS), followed by inva- 
sive ductal carcinoma (IDC) [18]. Thus, the presumptive precursor lesions, DH and DCIS represent an important model to study accumulation of chromosomal changes in the development of breast cancer.

Only few molecular studies have been performed on DH and DCIS of the breast to date [17]. Loss of heterozygosity $(\mathrm{LOH})$ within the p53 and the BRCA1 region, both located on chromosome 17 [15], has been described in patients with hyperplasia. Using immunohistochemistry and fluorescence in situ hybridization (FISH), c-erbB-2 overexpression was observed in DCIS and IDC but not in hyperplastic breast tissue [8].

Using comparative genomic hybridization ( $\mathrm{CGH}$ ), the entire genome can be investigated for DNA sequence copy number changes [13]. This technique has already been applied to the detection of chromosomal aberrations in breast cancer $[2,3,14,16,20,23,30]$, and a complex pattern of gains and losses has been found involving many chromosomes with DNA gains on 1q, 6p, 8q, 11q, 12q, 17q, 20q, and losses on $6 \mathrm{q}$ and $12 q[2,14,16,23]$. In addition, a distinct heterogeneity within infiltrating lesions has been demonstrated [2]. Recently, CGH studies have also been reported on DCIS $[3,12,16]$, and these have demonstrated a wide variety of chromosomal imbalances similar to those of IDC. These studies suggested that alterations in DCIS closely resemble those previously detected and described in IDC, corroborating the precursor status of intraductal carcinoma of the breast. Until now, ductal hyperplasias have not been extensively analysed by $\mathrm{CGH}$. The $20 \mathrm{q}$ amplification, which is thought to harbour a novel oncogene and may possibly give prognostic hint in invasive breast carcinoma $[1,26]$ was reported being amplified also in simple ductal hyperplasias [33]. In this report, DH are for the first time more extensively investigated by $\mathrm{CGH}$ to search for possible heterogeneity.

\section{Material and methods}

\subsection{Patients and tissue}

Formalin-fixed, paraffin-embedded tissue sections from 4 patients were used. Two cases had ductal hyperplasia (DH) and ductal carcinoma in situ (DCIS) adjacent to invasive ductal carcinoma (IDC). The two other cases had extensive DCIS adjacent to IDC. The cases were classified and subtyped according to standard criteria [5,24,25,31]. Histopathological diagnosis of $\mathrm{DH}$ were made by two pathologists
(M.C. and M.W.). Sequential $5 \mu \mathrm{m}$ sections were cut from the paraffin blocks, mounted on a coverslip and stained with hematoxylin and eosin (H\&E) for lasermicrodissection. Multiple samples were investigated from DH $(n=9)$ and DCIS $(n=12)$ (Table 1$)$.

\subsection{Microdissection, DOP-PCR and CGH}

A laser-based microdissection system (P.A.L.M., Wolfratshausen, Germany) was used to isolate histologically homogeneous cell groups from the different lesions, each consisting of 400 to 500 cells [3]. The samples were collected in a sterile tube, treated by proteinase $\mathrm{K}(100 \mathrm{mM}$ Tris $/ \mathrm{HCl}, 10 \mathrm{mg} / \mathrm{ml}$ proteinase $\mathrm{K}, \mathrm{pH}$ 7.5) [2,3], and DNA was amplified by degenerate oligonucleotide primed-polymerase chain reaction (DOP-PCR) to generate sufficient representative DNA [2,29,34]. Labeling of tumor DNA was performed with biotin-16-dUTP (Boehringer, Mannheim, Germany) using nick translation.

Metaphase slides were prepared from phytohemagglutinin-stimulated peripheral blood lymphocytes according to standard procedures [14]. CGH analysis was performed as previously described [14] with only slight modifications [3]. $500 \mathrm{ng}$ of the DNA from the tissue sample and $500 \mathrm{ng}$ SpectrumRed ${ }^{\mathrm{TM}}$ direct labeled normal female reference DNA (Vysis, Inc., Downers Grove, IL, USA) were hybridized together with $40 \mu \mathrm{g}$ Cot-1 DNA (Life Technologies Inc., Grand Island, NY, USA) on normal metaphase spreads. Detection of tumor DNA was performed with Cy2-conjugated streptavidin and biotinylated anti-streptavidin conjugates (Amersham Buchler, Braunschweig, Germany). Metaphase chromosomes were counterstained with DAPI $(0.05 \mu \mathrm{g} / \mathrm{ml})$.

Image aquisition and processing were performed as previously described [3,34] using a Zeiss Axioplan2 microscope (Zeiss Axiovert, Jena, Germany) and a digital image analysis system (MetaSystems, Altlussheim, Germany). From about 10 to 15 homologues of each chromosome average profiles of green to red fluorescence ratios were calculated. For interpretation of these profiles, statistical confidence intervals were calculated, and the width of the confidence intervals was determined using Student's $t$-test statistic $( \pm 3$ times standard deviation). Since artifactual results had been occasionally observed on chromosomal bands 1p34p36 and chromosome 19 [3,32], these regions were excluded from the interpretation. The most important test in our attempt was the repeated investigation of nontumorous tissue cells. The reliability of our complex 
method was verified in each patient with nontumorous microdissected cells, which all showed $\mathrm{CGH}$ profiles without any deviation from the normal range.

\section{Results}

Figures on http://www.esacp.org/acp/2000/20-1/ aubele.htm

The CGH results from the microdissected samples from DH, DCIS, and IDC of the four patients analysed are summarized in Table 1 with the minimal common region of chromosomal alterations shown. The DH samples from case one showed an average of 5.3 $( \pm 1.4$ SEM) chromosomal aberrations. In all DH samples gain of DNA was identified on chromsome 20q. In $3 / 4$ samples DNA gain was present on $6 \mathrm{p}$, and DNA loss on 13q (Table 1). Two of four samples showed gain of DNA on chromosomal regions 1q, 11q13, 14q and 16p. Except for gain of DNA on $6 p$ all of these abnormalities were also present in the adjacent DCIS samples. In addition to the abnormalities found in $\mathrm{DH}$, all 3 DCIS samples showed DNA loss on chromosome $6 \mathrm{q}$ as well as gain on $15 \mathrm{q}$. Here, in $2 / 3$ samples additional alterations were identified on chromosomes $3 p$ and 10q. Some heterogeneity in the CGH results, however, was evident within samples from both $\mathrm{DH}$ and DCIS. This heterogeneity was mostly demonstrated in the DCIS samples in chromosomal region $9 \mathrm{p}$, and in the DH samples in chromosomal regions $11 \mathrm{q} 13,14 \mathrm{q}$ and 16p (Fig. 1). The chromosomal changes we identified in DCIS were also found in the adjacent IDC. Remarkably, gain of DNA on chromosome $6 p$ was found in 3/4 DH samples from case one, however, was not identified in the DCIS or IDC samples (Table 1).

In case two an average of 5.8 ( \pm 1.3 SEM) chromosomal imbalances was identified in the $5 \mathrm{DH}$ samples. In at least $2 / 5$ samples gain of DNA was observed on chromosomes 3p, 6p, 8q, 10q, 15q, 16p, 17 and 20q. Chromosomal losses were identified on $4 q$ and 13q. Representative examples from CGH profiles demonstrating heterogeneity are given in Fig. 2A. The only aberration present in each of the five $\mathrm{DH}$ samples was also amplification on chromosome 20q (Fig. 2B). All of the aberrations found in $\mathrm{DH}$ were also identified in the adjacent DCIS samples, however, with increased constancy. The additional chromosomal changes in DCIS were $3 q+, 5 q-, 8 p-, 9 q+/ 9 p-$, $11 \mathrm{q} 13+($ Table 1$)$. The abnormalities in DCIS corresponded mainly to those in the adjacent IDC.

In the two cases of extensive DCIS adjacent to small infiltrating lesions (IDC) three representative DCIS samples were investigated together with IDC. Abnormalities most consistently found were DNA gains on chromosomes 1q, 8q, 10q, 16p, 17q and 20q, and losses on chromosomes $9 \mathrm{p}$ and $13 \mathrm{q}$. Here, heterogeneous CGH results were mostly demonstrated on chromosomes 6, 8, 12, 16 and 17 (Fig. 3).

Alltogether, the breast tissue investigated here by CGH gave an average of $5.6( \pm 0.9)$ abnormalities in nine DH samples, $10.8( \pm 0.9)$ in the twelve DCIS samples, and $14.8( \pm 0.9)$ in IDC. In Table 1, all samples from one patient and from identical entities all showing the same chromosomal alteration are highlighted.

\section{Discussion}

In this study we have identified a great range of chromosomal changes in preneoplastic and neoplastic breast tissue. Although the number of cases presented here is low, the 12 DCIS and 9 DH samples from our four patients produced clear evidence, that heterogeneous chromosomal imbalances are present within a single histopathological entity from an individual patient. This heterogeneity was most pronounced within samples from DH. One possible explanation for our heterogeneous findings may be that multiple clones with varying chromosomal changes exist already in the DHs. Multiclonality may also explain that some alterations we identified in the DH samples were not present in the DCIS samples from the same patient, probably due to sampling of clones in $\mathrm{DH}$, which were not sampled in the corresponding DCIS. A second possible explanation might be that clonal selection took place during the progression from DH to DCIS. Studies of conventional cytogenetic analysis had already reported, that cytogenetically unrelated clones are present within one tumor, and that these are a feature of both in situ carcinoma and invasive breast lesions [11, 30]. All these findings possibly signify that, at least in the cases investigated here, a tumorigenesis pathway aside from a simple linear model should be considered.

Matching results between reported chromosomal changes and our findings in invasive breast carcinomas were mainly gains on $20 q[11,14,23], 8 q$ and $17 q[14$, $23,16]$, and loss on $13 q[11,16]$. The most consistent chromosomal imbalance we found in our samples was amplification of the $20 q 13$ region, which has already been described as one of the most frequent abnormalities in invasive breast cancer studies $[2,3,11,14,16,18$, 23]. In DCIS, however, CGH results so far are controversial as $20 q$ amplification was not identified in the 
Summary of investigated samples, histopathological diagnosis, and CGH results with the minimal common region of alteration. Samples from one patient and identical entities all showing the same chromosomal aberration are highlighted (*)

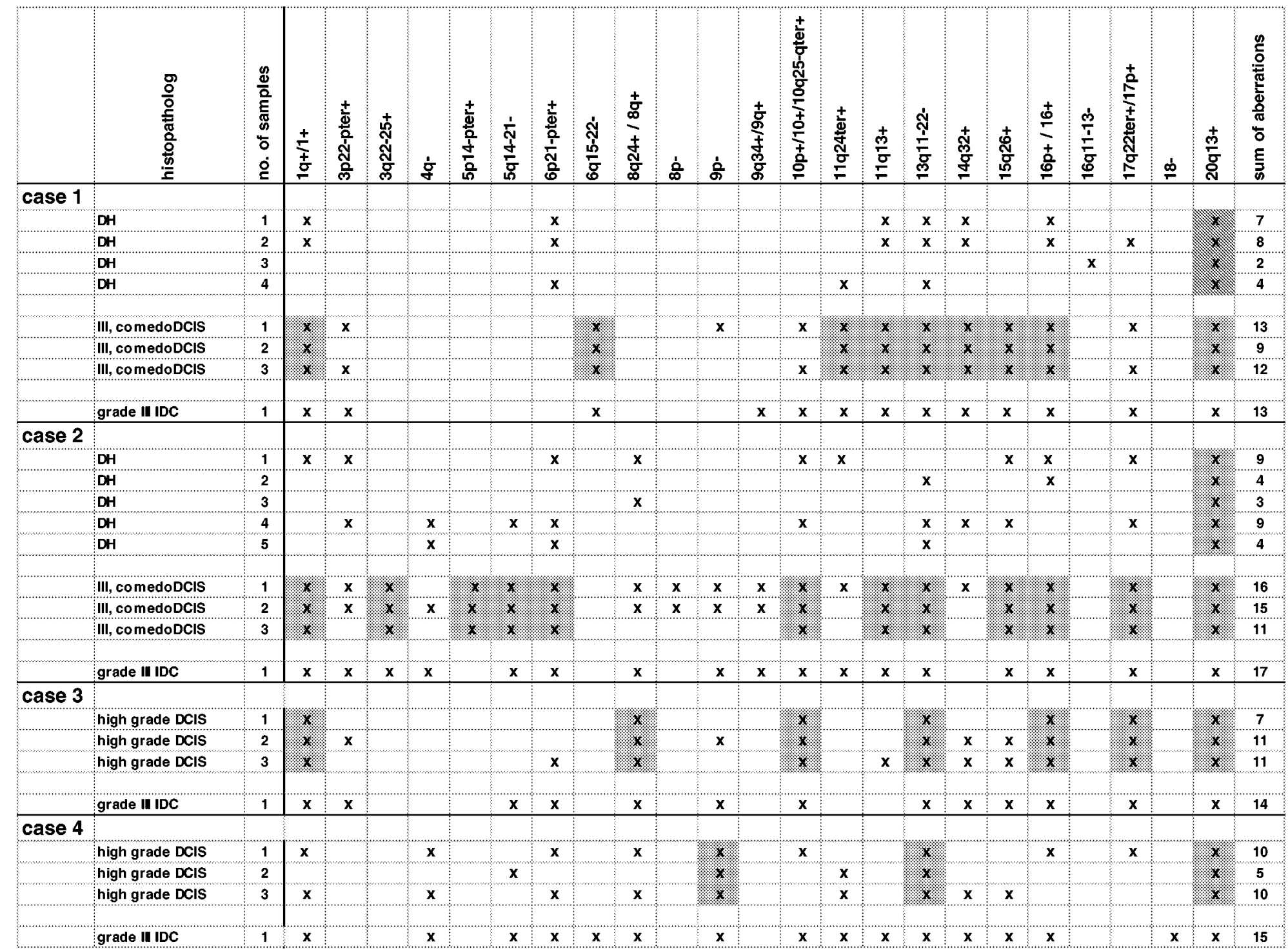


DH 1

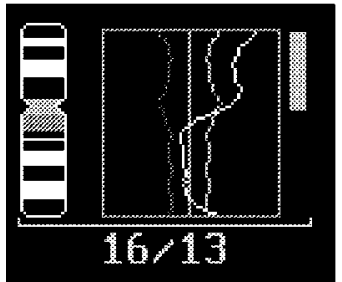

DH 2

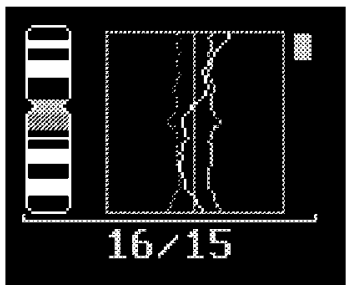

DH 3

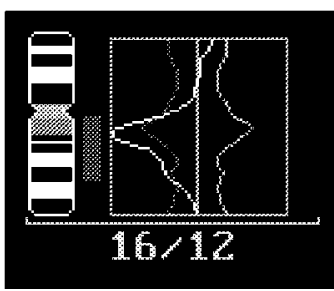

DH 4

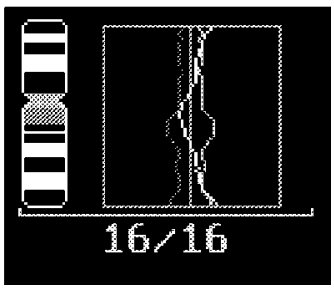

Fig. 1. Averaged CGH profiles from case 1. The confidence intervals ( \pm 3 times standard deviations) are plotted (red and green) together with the average ratio profile (white lines). Red bars are indicative for loss, green bars for gain of DNA. Given is also the number of the chromosome and the number of selected homologues. Heterogeneity in four DH samples is demonstrated by chromosome 16.

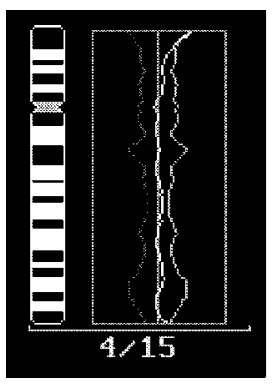

DH 1

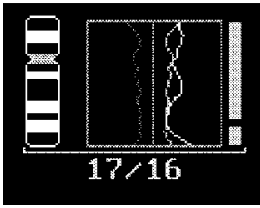

DH 1

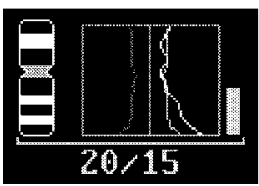

DCIS 1

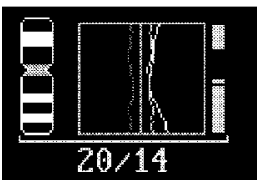

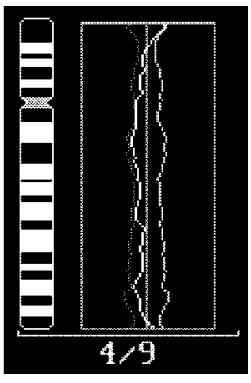

DH 2

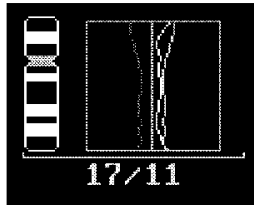

DH 2

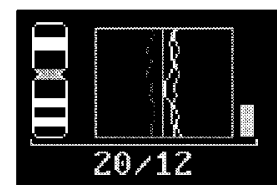

DCIS 2

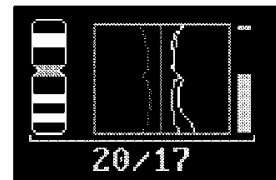

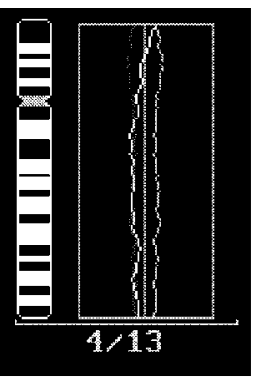

DH 3

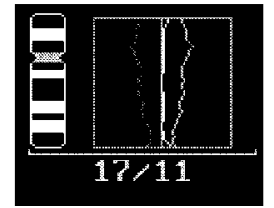

DH 3

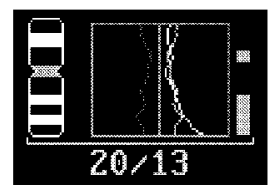

DCIS 3

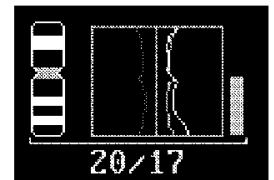

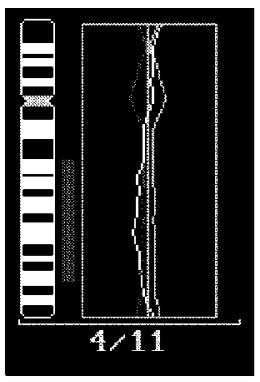

DH 4

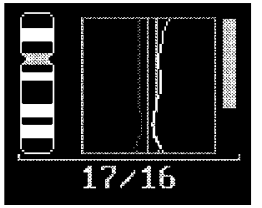

DH 4

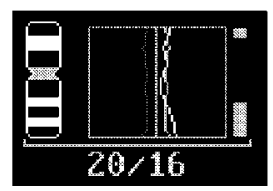

IDC

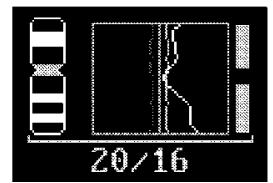

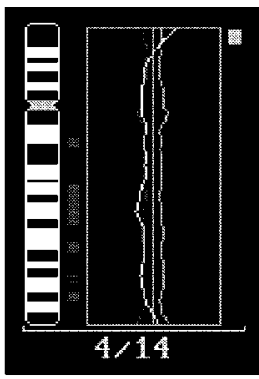

DH 5

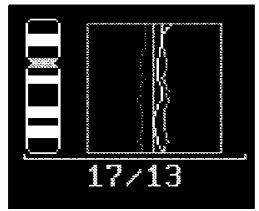

DH 5

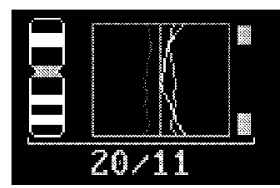

$\mathrm{N}$

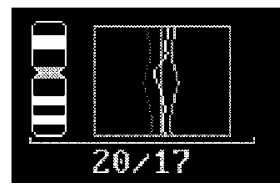

Fig. 2. Representative examples of CGH profiles from case 2. (A) Heterogeneity in CGH profiles demonstrated by chromosomes 4 and 17. (B) Examples for the most consistent chromosomal gain on chromosome 20. Averaged profiles are given for chromosome 20 from all samples of case 2, including 5 DH, 3 DCIS, the IDC sample, as well as the sample from the normal cells.

CIS lesions studied by Kuukasjärvi et al. [16], and in only a minority of CIS lesions by Buerger et al. [6]. We have identified this alteration with high frequency in high grade DCIS [3], and have shown that this amplification is already present in $\mathrm{DH}$ lesions adjacent to DCIS and IDC [33]. The 20q13 region is thought to 


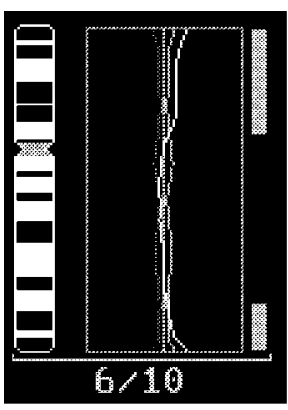

DCIS 1

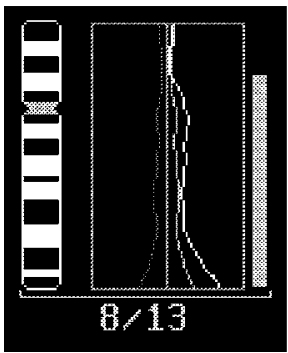

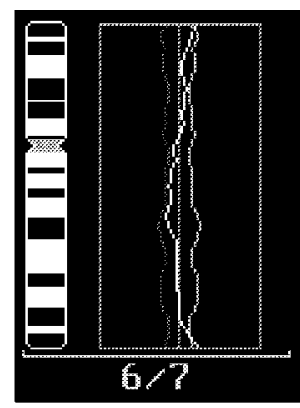

DCIS 2

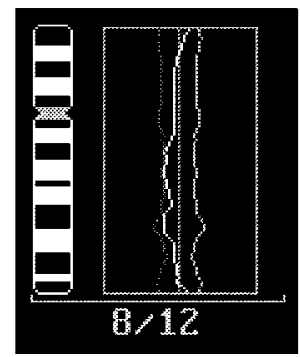

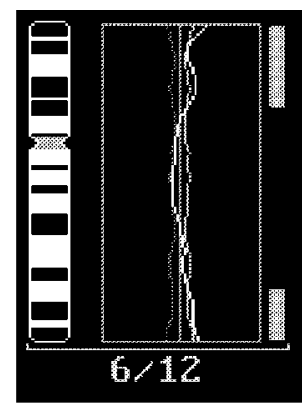

DCIS 3

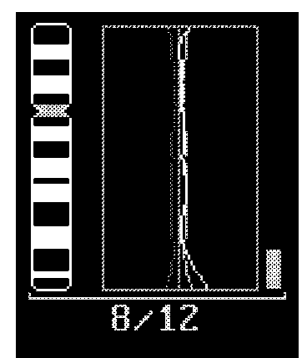

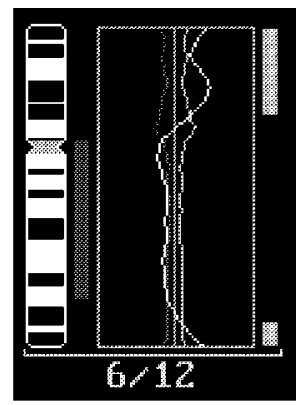

IDC

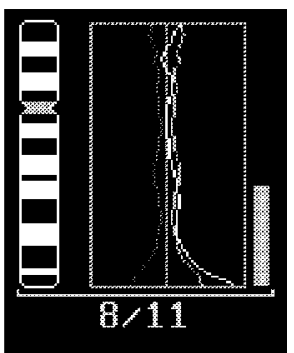

Fig. 3. Examples for heterogeneous CGH results from 3 DCIS and one IDC sample from case 4. Given are profiles from chromosome 6 and 8.

harbour a novel oncogene termed AIB (amplified in breast cancer) [1,26], however, so far, no potential candidate gene has been identified in this chromosomal region. That this gain of DNA was present consistently with even the earliest morphological changes, supports its important role in breast carcinogenesis.

DNA gain on chromosome 1 was identified in nearly all of our DCIS samples, but only in a minority of DH samples. Amplification of chromosome 1 was also identified by CGH in DCIS $[3,16]$, in all cytometrically diploid as well as in $50 \%$ of the aneuploid breast carcinomas [23], and by FISH analysis in ductal hyperplasias [9]. In DCIS, amplification of the chromosomal region $17 q$ (17q21, harbouring erbB2 oncogene) as well as polysomy $17[16,20,30]$ was found, and erbB2 protein overexpresion has been identified in a high proportion of high nuclear grade DCIS, but is uncommon in the low grade forms [17].

DNA gain on chromosome $6 \mathrm{p}$, harbouring several potential candidate genes, e.g., PIM1 (pim-1oncogene), and E2F3 (E2F transcription factor 3) was consistently found in our DCIS samples. In the DH samples, as with several other chromosomes, distinct heterogeneity was demonstrated.

Gain on chromosomal region 11q13 was found in case 1 in 2/4 DH samples, in all of the DCIS and in the one IDC sample. In case 2, however, none of the DH samples but all three DCIS samples also showed this amplification. Gain on 11q13 was also found by Tanner et al. [27] in hypodiploid breast cancers. The authors furthermore demonstrated, that Cyclin D1 oncogene was affected by this amplification. In ductal hyperplasias, amplification of $11 \mathrm{q} 13$ was detected only at low levels [4].

The second most frequent alteration in our study was loss on chromosome $13 q$. Loss on $13 q$ has been reported to occur frequently in IDC [20,22] and in DCIS [3]. The smallest commonly deleted region was 13q11-22, including both Rb1 and BRCA2.

According to the multistep model of breast carcinogenesis $[17,19]$, tumors may develop and progress as a consequence of alterations in oncogene and tumorsuppressor gene loci [7]. As there is no detailed molecular model of the critical genetic events in breast cancer [7], the role of the presumptive precursor lesions DH and DCIS in the progression pathway needs to be identified. The study presented here reports CGH results from multiple samples of potential precursor lesions, microdissected from breast tissue of four patients. Our results clearly demonstrate that heterogeneous chromosomal imbalances are present within DH and DCIS of a patient. This heterogeneity was most expressed within samples from DH. However, the more consistently identified alterations such as $20 \mathrm{q}$ or $13 \mathrm{q}$, present in most samples of DH, suggest an important role of genes localized in these chromosomal regions for breast carcinogenesis. 


\section{Acknowledgment}

The authors gratefully acknowledge the excellent technical assistance of Daniela Angermaier and Ilse Di Grazia. This work was supported by the Wilhelm Sander - Stiftung (96.07.01/2), München, Germany.

\section{References}

[1] S.L. Anzick, J. Kononen, R.L. Walker, D.O. Azorsa, M.M. Tanner, X.-Y. Guan, G. Sauter, O.-P. Kallioniemi, J.M. Trent and P.S. Meltzer, AIB1, a steroid receptor coactivator amplified in breast and ovarian cancer, Science 277 (1997), 965-968.

[2] M. Aubele, A. Mattis, H. Zitzelsberger, A. Walch, M. Kremer, P. Hutzler, H. Höfler and M. Werner, Intratumoral heterogeneity in breast carcinoma revealed by laser-microdissection and comparative genomic hybridization (CGH), Cancer Gen. Cytogen. 110 (1999), 94-102.

[3] M. Aubele, A. Mattis, H. Zitzelsberger, A. Walch, M. Kremer, G. Welzl, H. Höfler and M. Werner, Extensive ductal carcinoma in situ with small foci of invasive ductal carcinoma: Evidence of genetic resemblance by CGH, Int. J. Cancer 85 (2000), 8286.

[4] M.W. Beckmann, D. Niederacher, H.G. Schnürch, B.A. Gusterson and H.G. Bender, Multistep carcinogenesis of breast cancer and tumor heterogeneity, J. Mol. Med. 75 (1997), 429-439.

[5] H.J. Bloom and W.W. Richardson, Histological grading and prognosis in breast cancer. A study of 1049 cases of which 359 have been followed for 15 years, Br. J. Cancer 11 (1957), 359377.

[6] H. Buerger, F. Otterbach, R. Simon, C. Poremba, R. Diallo, T. Decker, L. Riethdorf, C. Brinkschmidt, B. DockhornDworniczak and W. Boecker, Comparative genomic hybridization of ductal carcinoma in situ of the breast-evidence of multiple genetic pathways, J. Pathol. 187 (1999), 396-402.

[7] S.A. Chappell, T. Walsh, R.A. Walker and J.A. Shaw, Loss of heterocygocity at chromosome $6 \mathrm{q}$ in preinvasive and early invasive breast carcinomas, Br. J. Cancer 75 (1997), 1324-1329.

[8] E.D. Coene, V. Schelfhout, R.A. Winkler, A.-M. Schelfhaut, N. van Roy, M. Grooteclaes, F. Speleman and C.R. de Potter, Amplification units and translocation at chromosome 17q and c-erbB-2 overexpression in the pathogenesis of breast cancer, Virchows Arch. 430 (1997), 365-372.

[9] M.C. Cummings, M. Aubele, A. Mattis, D. Purdie, P. Hutzler, H. Höfler and M. Werner, Increasing chromosome 1 copy number parallels histological progression in breast carcinogenesis, Br. J. Cancer 82 (2000), 1204-1210.

[10] S. Heim, M.R. Teixeira, C.U. Dietrich and N. Pandis, Cytogenetic polyclonality in tumors of the breast, Cancer Genet. $C y$ togenet. 95 (1997), 16-19.

[11] J.J. Isola, O.-P. Kallioniemi, L.W. Chu et al., Genetic aberrations detected by comparative genomic hybridization predict outcome in node-negative breast cancer, Am. J. Pathol. 147 (1995), 905-911.
[12] L.A. James, E.L.D. Mitchell, L. Menasce and J.M. Varley, Comparative genomic hybridisation of ductal carcinoma in situ of the breast: identification of regions of DNA amplification and deletion in common with invasive breast carcinoma, Oncogene 14 (1997), 1059-1065.

[13] A. Kallioniemi, O.-P. Kallioniemi, D. Sudar, D. Rutovutz, J.W. Gray, F. Waldman and D. Pinkel, Comparative genomic hybridization for molecular cytogenetic analysis of solid tumors, Science 258 (1992), 818-821.

[14] A. Kallioniemi, O.-P. Kallioniemi, J. Piper, M. Tanner, T. Stokke, L. Chen, H.S. Smith, D. Pinkel, J.W. Gray and F.M. Waldman, Detection and mapping of amplified DNA sequences in breast cancer by comparative genomic hybridization, Proc. Natl. Acad. Sci. USA 91 (1994), 2156-2160.

[15] M. Kasami, C.L. Vnencak-Jones, S. Manning, W.D. Dupont and D.L. Page, Loss of heterozygosity and microsatellite instability in breast hyperplasia, Am. J. Pathol. 150 (1997), 19251932.

[16] T. Kuukasjärvi, R. Karhu, M. Tanner, M. Kahkonen, A. Schaffer, N. Nupponen, S. Pennanen, A. Kallioniemi, O.-P. Kallioniemi and J. Isola, Genetic heterogeneity and clonal evolution underlying development of asynchronous metastasis in human breast cancer, Cancer Res. 57 (1997), 1597-1604.

[17] S.R. Lakhani, The transition from hyperplasia to invasive carcinoma of the breast, J. Pathol. 187 (1999), 272-278.

[18] E. Moore, H. Magee, J. Coyne, T. Gorey and P.A. Dervan, Widespread chromosomal abnormalities in high-grade ductal carcinoma in situ of the breast. Comparative genomic hybridization study of pure high-grade DCIS, J. Pathol. 187 (1999), 403-409.

[19] I.F. Newsham, The long and short of chromosome 11 in breast cancer, Am. J. Pathol. 153 (1998), 5-9.

[20] T. Nishizaki, S. DeVries, K. Chew, W.H. Goodson, B.M. Ljung, A. Thor and F.M. Waldman, Genetic alterations in primary breast cancers and their metastases: direct comparison using modified comparative genomic hybridization, Genes Chromos. Cancer 19 (1997), 267-272.

[21] D.L. Page, W.D. Dupont, L.W. Rogers and M.S. Rados, Atypical hyperplastic lesions of the female breast. A long-term follow-up study, Cancer 55 (1985), 2698-2708.

[22] D.M. Radford, K.L. Fair, N.J. Phillips, J.H. Ritter, T. Steinbrueck, M.S. Holt and H. Donis-Keller, Allelotyping of ductal carcinoma in situ of the breast: deletion of loci on 8p, 13q, 16q, 17p, 17q, Cancer Res. 55 (1995), 3399-3405.

[23] T. Ried, K.E. Just, H. Holtgreve-Grez, S. duManoir, M.R. Speicher, E. Schröck, C. Latham, H. Blegen, A. Zetterberg, T. Cremer and G. Auer, Comparative genomic hybridization of formalin-fixed, paraffin-embedded breast tumors revealed different patterns of chromosomal gains and losses in fibroadenomas and diploid and aneuploid carcinomas, Cancer Res. 15 (1995), 5415-5420.

[24] P.P. Rosen, H.A. Oberman and AFIP (Armed Forces Institute of Pathology), Tumors of the Mammary Gland, 3rd edn, Washington, DC, 1993.

[25] M.J. Silverstein, D.N. Poller and J.R. Walsman, Prognostic classification of breast ductal carcinoma-in-situ, The Lancet 345 (1995), 1154-1157. 
[26] M.M. Tanner, M. Tirkkonen, A. Kallioniemi, C. Collins, T. Stokke, R. Karhu, D. Kowbel, F. Shadravan, M. Hintz, W.L. Kuo, F.M. Waldman, J.J. Isola, J.W. Gray and O.-P. Kallioniemi, Increased copy number at 20q13 in breast cancer: defining the critical region and exclusion of candidate genes, Cancer Res. 54 (1994), 4257-4260.

[27] M.M. Tanner, R.A. Karhu, N.N. Nupponen, A. Borg, B. Baldetorp, T. Pejovic, M. Ferno, D. Killander and J.J. Isola, Genetic aberrations in hypodiploid breast cancer: frequent loss of chromosome 4 and amplification of cyclin D1 oncogene, Am. J. Pathol. 153 (1998), 191-199.

[28] M.R.Teixeira, N. Pandis, G. Bardi, J.A. Andersen, F. Mitelman and S. Heim, Clonal heterogeneity in breast cancer: karyotypic comparison of multiple intra- and extra-tumorous samples from 3 patients, Int. J. Cancer 63 (1995), 63-68.

[29] H. Telenius, N.C. Carter, C.E. Bebb, M. Nordenskjöld, B.A.J. Ponder and A. Tunnacliffe, Degenerate oligonucleotideprimed PCR: general amplification of target DNA by a single degenerate primer, Genomics 13 (1992), 718-725.

[30] M. Tirkkonen, M. Tanner, R. Karhu, A. Kallioniemi, J. Isola and O.-P. Kallioniemi, Molecular cytogenetics of primary breast cancer by CGH, Genes, Chromos Cancer 21 (1998), 177-184.
[31] UICC (International Union against Cancer), Classification of Malignant Tumors, L.H. Sobin and C. Wittekind, eds, WileyLiss, New York, 1997.

[32] R.G. Weber, M. Scheer, I.A. Born, S. Joos, J.M.J.L. Cobbers, C. Hofele, G. Reifenberger, J.E. Zöller and P. Lichter, Recurrent chromosomal imbalances detected in biopsy material from oral premalignant and malignant lesions by combined tissue microdissection, universal DNA amplification, and comparative genomic hybridization, Am. J. Pathol. 153 (1998), 295303.

[33] M. Werner, A. Mattis, M. Aubele, M. Cummings, P. Hutzler and $\mathrm{H}$. Höfler, 20q13.2 amplification in intraductal hyperplasia adjacent to in situ and invasive cancer of the breast, Virchows Arch. 435 (1999), 469-472.

[34] H. Zitzelsberger, U. Kulka, L. Lehmann, A. Walch, J. Smida, M. Aubele, T. Lörch, H. Höfler, M. Bauchinger and M. Werner, Genetic heterogeneity in a prostatic carcinoma and associated prostatic intraepithelial neoplasia as demonstrated by combined use of laser-microdissection, degenerate oligonucleotide primed PCR and comparative genomic hybridization, Virchows Arch. 433 (1998), 297-304. 


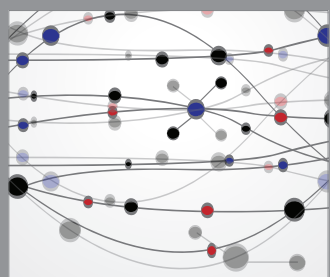

The Scientific World Journal
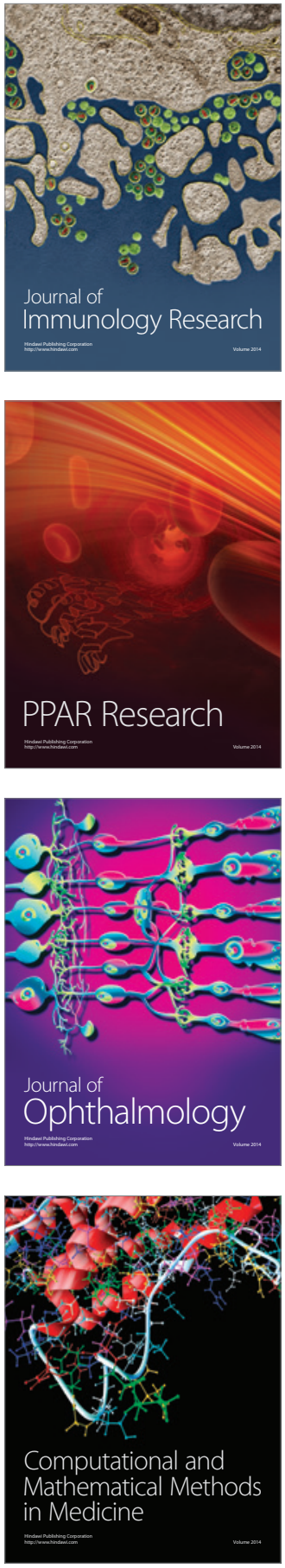

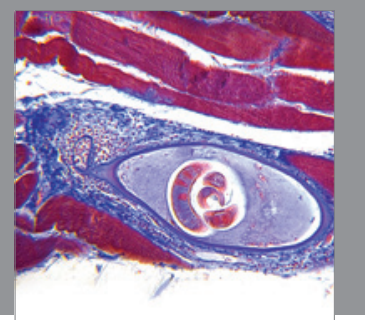

Gastroenterology

Research and Practice
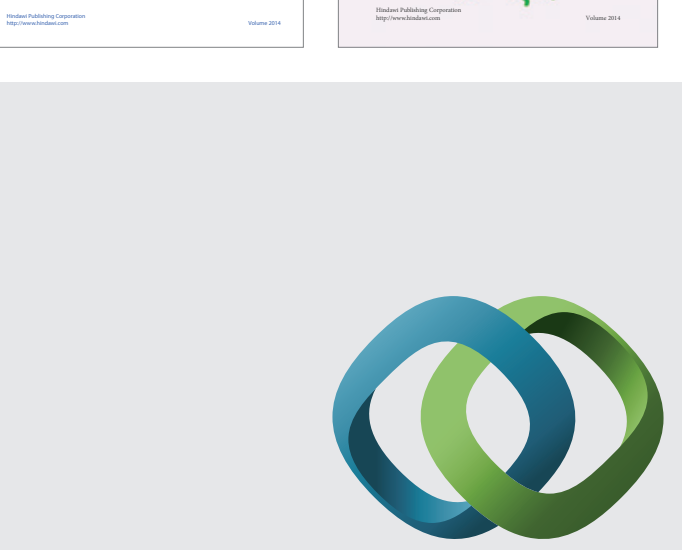

\section{Hindawi}

Submit your manuscripts at

http://www.hindawi.com
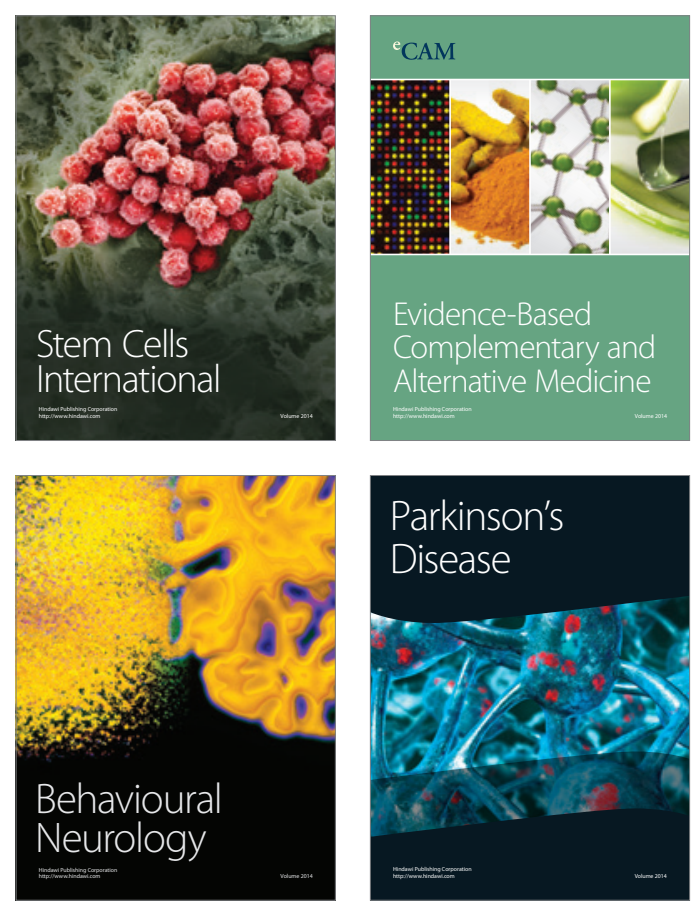

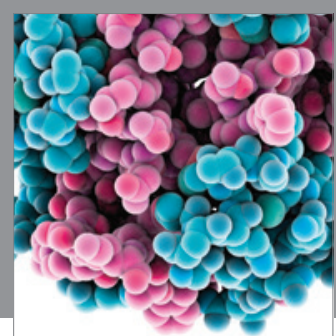

Journal of
Diabetes Research

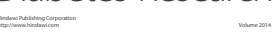

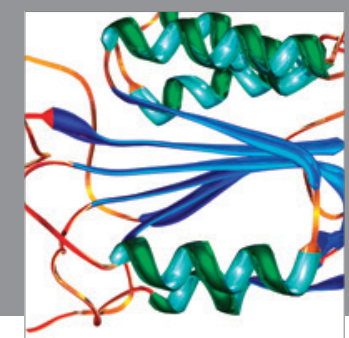

Disease Markers
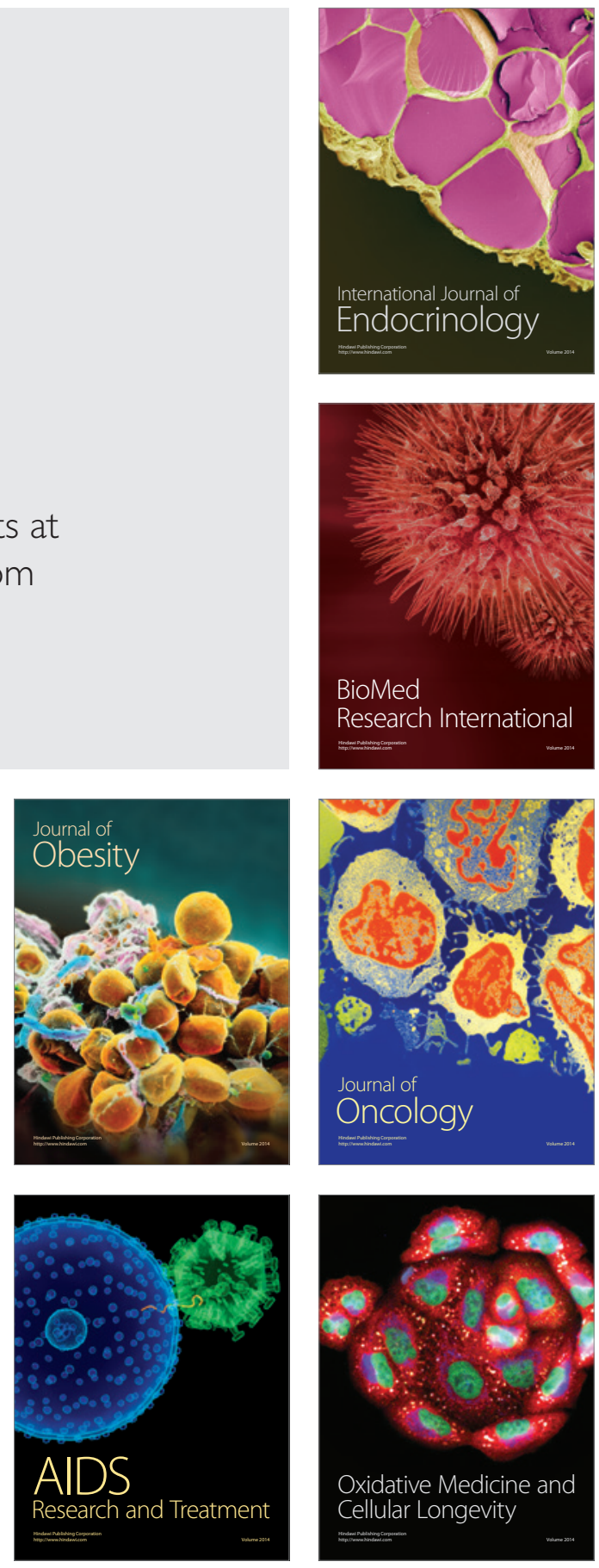